Int. J. Odontostomat., 7(2):293-297, 2013.

\title{
Manejo de Herida Quirúrgica en Infección del Territorio Maxilofacial
}

\author{
Management of Surgical Wound Infection of Maxillofacial Area
}

Valenzuela, A., ${ }^{*, *} ;$ Raposo, A.,***; Preisler, G., ${ }^{*, *} \&$ Salinas, F.*

\begin{abstract}
VALENZUELA, A.; RAPOSO, A.; PREISLER, G. \& SALINAS, F. Manejo de herida quirúrgica en infección del territorio maxilofacial. Int. J. Odontostomat., 7(2):293-297, 2013.

RESUMEN: EI manejo de infecciones maxilofaciales leves o graves tiene como objetivos generales la protección de la vía aérea, eliminación quirúrgica de la fuente de infección, drenajes quirúrgicos de la lesión, antibioterapia adecuada, control de evolución de la lesión y herida facial hasta la resolución completa del proceso infeccioso. El objetivo de este artículo es presentar un caso clínico de una infección del territorio maxilofacial, de difícil manejo local y su respuesta al tratamiento junto a la evolución y manejo de la herida facial resultante. Paciente sexo masculino de 48 años de edad, sin antecedentes mórbidos relevantes se presenta en el servicio de Urgencias del Hospital Base de Valdivia con el diagnóstico de flegmón facial izquierdo. Evoluciona con resistencia al tratamiento convencional y lenta cicatrización de herida quirúrgica posterior al vaciamiento del flegmón abscedado. Se utiliza un hidrogel como coadyudante a la terapia, resultando finalmente en la resolución del cuadro clínico. Se discute evolución del cuadro infeccioso junto a las diferentes terapias coadyuvantes para un proceso infeccioso severo.
\end{abstract}

PALABRAS CLAVE: infección maxilofacial, cicatrización de heridas, apósito para heridas, hidrogel.

\section{INTRODUCCIÓN}

Las infecciones del territorio maxilofacial que afectan a la cara y el cuello presentan reacciones inflamatorias severas. Dentro de las cuales podemos encontrar, según su origen, las de tipo odontogénico, sinusal, glandular, traumático y origen dérmico (Wang et al., 2012), siendo frecuentes; los abscesos peritonsilares, necrosis pulpar dentaria, fracturas de huesos faciales, complicaciones postoperatorias de una herida facial, quistes infectados, inflamación de los ganglios linfáticos, afecciones inflamatorias del hueso mastoides, lesiones de la pared posterior de la faringe, complicaciones de tonsilectomía, infección de glándulas salivales y en ocasiones la causa no se identifica (Nienartowicz et al., 2008). El tratamiento convencional para este tipo de infecciones consiste en terapias antibióticas, cirugía y drenaje de flujo de la zona del cuello. También podemos encontrar tratamientos adyuvantes, especialmente de inmunoglobulina (Tejeda et al., 1999).

Para el manejo de las heridas resultantes posteriores a un proceso infeccioso severo, se siguen utilizando las suturas, las bandas adhesivas, mallas regeneradoras entre otras, buscando siempre lograr los mejores resultados estéticos, con la mínima incidencia de dehiscencias y/o reinfecciones (Guerra et al., 2005). En este caso se realizó un manejo de la herida con aseos quirúrgicos, apósitos y el uso de hidrogel local, gel utilizado en el relleno de heridas en forma primaria, en heridas agudas superficiales o en heridas crónicas para promover la degradación autolítica y la granulación (Ramirez \& Dagnino, 2006).

\section{CASO CLÍNICO}

Paciente sexo masculino de 48 Años de edad, sin antecedentes mórbidos de relevancia, se presenta al servicio de urgencias del Hospital Base de Valdivia en noviembre del año 2011. Paciente presentaba asimetría facial por aumento de volumen geniano, cuerpo mandibular y región cervical izquierda compatible con flegmón facial, se observa la piel con consistencia firme y turgente (Fig. 1).

\footnotetext{
* Departamento de Cirugía Buco-maxilofacial, Universidad Austral de Chile, Valdivia, Chile.

${ }^{* *}$ Servicio de Cirugía Buco-maxilofacial Hospital Base de Valdivia, Valdivia, Chile.
} 


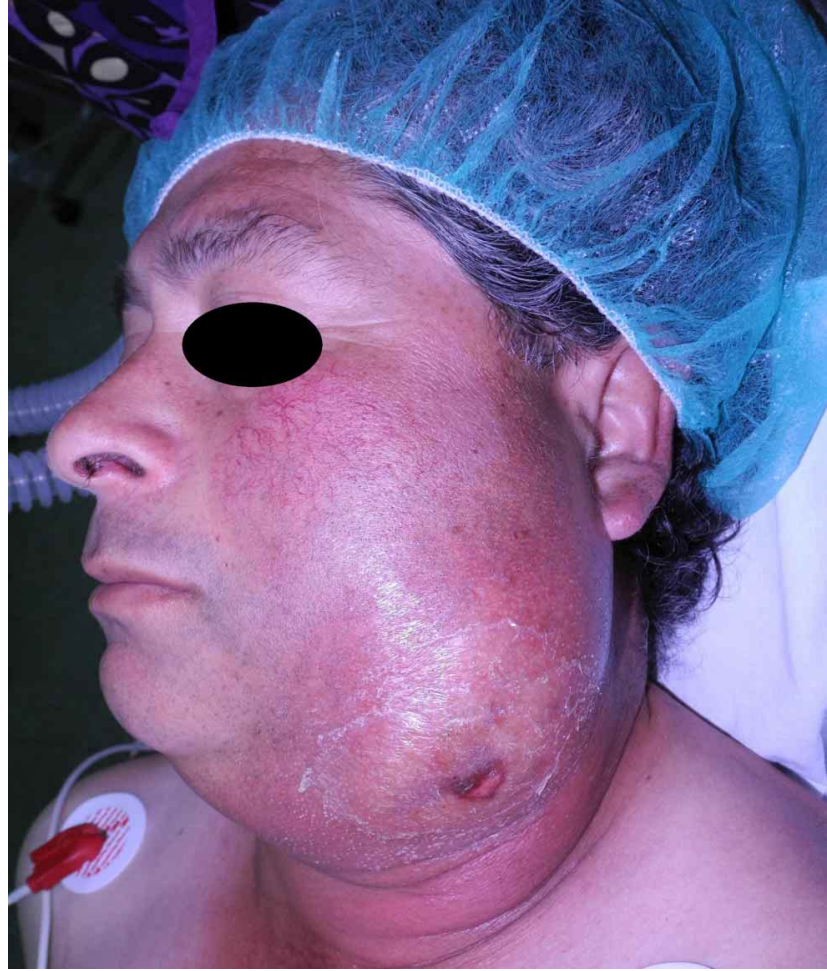

Fig. 1. Paciente con el flegmón facial previo a la cirugía.

Al examen intraoral presenta una lesión ulcerada en mucosa oral por impronta del diente 2.8. Encontrándose también la presencia de caries dentinaria profunda en el diente 3.6. Paciente queda hospitalizado con el siguiente esquema; clindamicina $600 \mathrm{mg}$ EV cada $8 \mathrm{~h}$, ketorolaco $30 \mathrm{mg} \mathrm{EV}$ cada $8 \mathrm{~h}$, ranitidina $50 \mathrm{mg}$ EV cada $8 \mathrm{~h}$, suero fisiológico $1500 \mathrm{cc}$ en $24 \mathrm{~h}$, aseo bucal y enjuagues con clorhexidina $0,12 \%$. Al segundo día el paciente fue sometido a cirugía consistente en el vaciamiento e instalación de un drenaje penrose (Fig. 2).

Al cuarto día el paciente se mantiene con apósito en la zona de la lesión, presentando a la palpación el tejido menos indurado en la región cervical, y se observa salida de material purulento por el penrose.

Se irriga con suero fisiológico y se instala el apósito nuevamente se mantiene la misma terapia farmacológica. Se solicitan exámenes sanguíneos para verificar evolución del cuadro. Al quinto día se encuentra en condiciones similares mejorando parámetros sanguíneos, drenando esporádicamente exudado purulento por dentro de la boca. Al sexto día presenta secreción purulenta y abundante tejido necrótico en la lesión (Fig. 3).

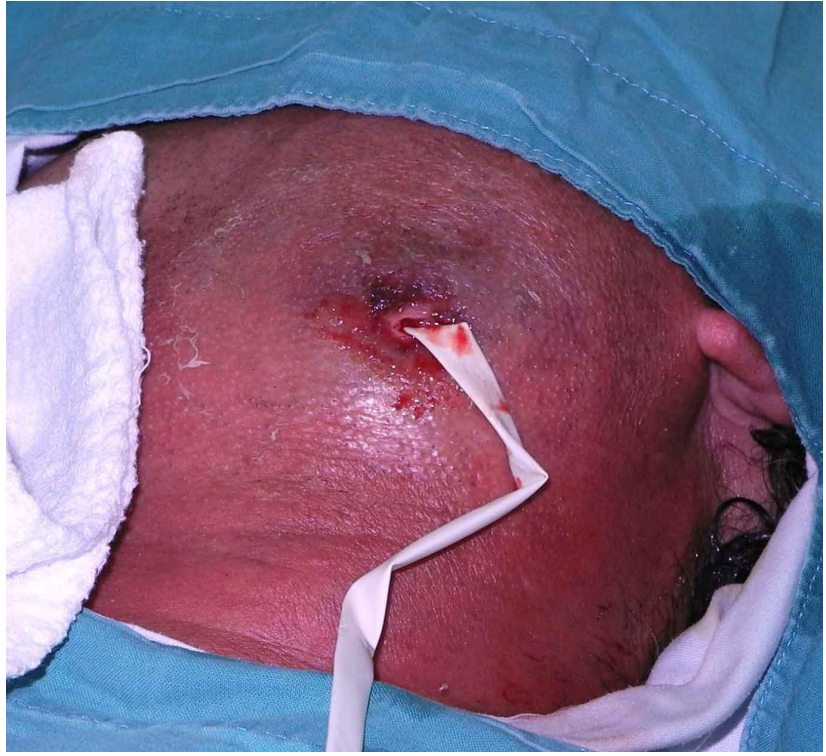

Fig. 2. Instalación del sistema de drenaje Penrose.

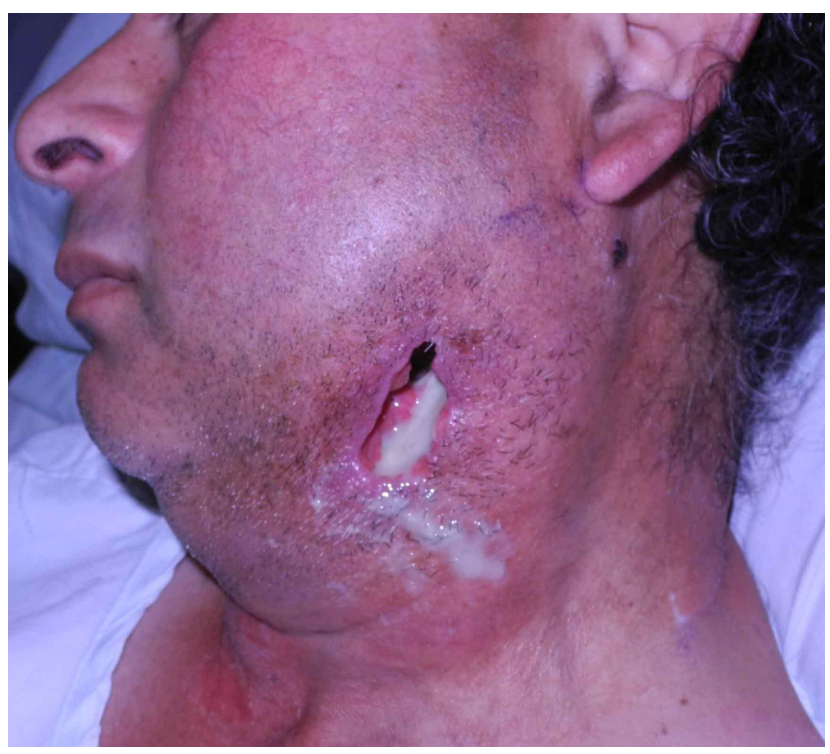

Fig. 3. Paciente presenta exudado purulento y presencia de tejido necrótico en fondo de la lesión.

Se refiere interconsulta a infectólogo para evaluar presencia de cepas resistentes. El infectólogo indica aumentar frecuencia de las dosis de clindamicina a 600 mg cada $6 \mathrm{~h}$. El resultado del cultivo mostró la presencia de 2 microorganismos predominantes, el Staphylococus epidermidis y el Enterobacter cloacae ssp cloacae. Al día 8 evoluciona la herida con formación de tejido de granulación en el fondo. Se mantienen indicaciones del infectólogo. Al día 14 posterior a su ingreso al hospital en la unidad de cirugía maxilofacial se realizó las exodoncias de los dientes 2.8 y 3.6 (posibles focos del cuadro). Se decide apli- 
car terapia coadyuvante al manejo de la herida con la aplicación de hidrogel local, gel utilizado para fomentar la degradación autolítica y la formación de tejido de granulación. El tratamiento con el hidrogel se realizó con una aplicación diaria los primeros 4 días, disminuyendo su frecuencia al constatar eficacia en la eliminación del tejido necrótico. Se disminuye la frecuencia de aplicación a una cada 3 días cuando se produjo la eliminación del tejido necrótico y la correcta evolución de la cicatrización (Fig. 4), Se suspende tratamiento endovenoso y se reemplaza por tratamiento vía oral dado la evolución favorable del cuadro, quedando con las siguientes indicaciones; clindamicina $300 \mathrm{mg} \mathrm{c} / 8 \mathrm{hr}$, ranitidina $150 \mathrm{mg} \mathrm{c} / 12 \mathrm{~h}$, diclofenaco sódico $50 \mathrm{mg} \mathrm{c} / 8 \mathrm{~h}$, Higiene bucal con clorhexidina $0,12 \%$ y cambio del apósito cada $6 \mathrm{~h}$.

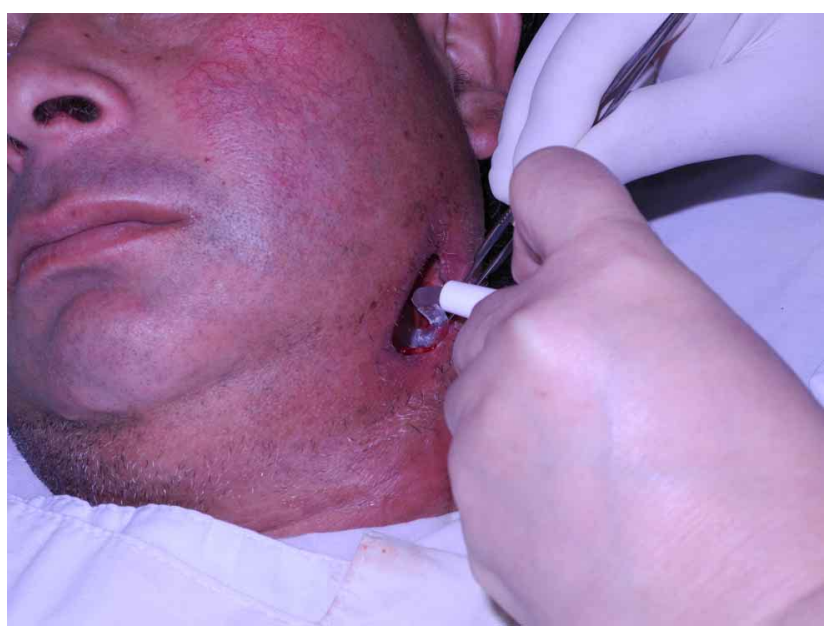

Fig. 4. Aplicación de hidrogel en fondo de la herida.

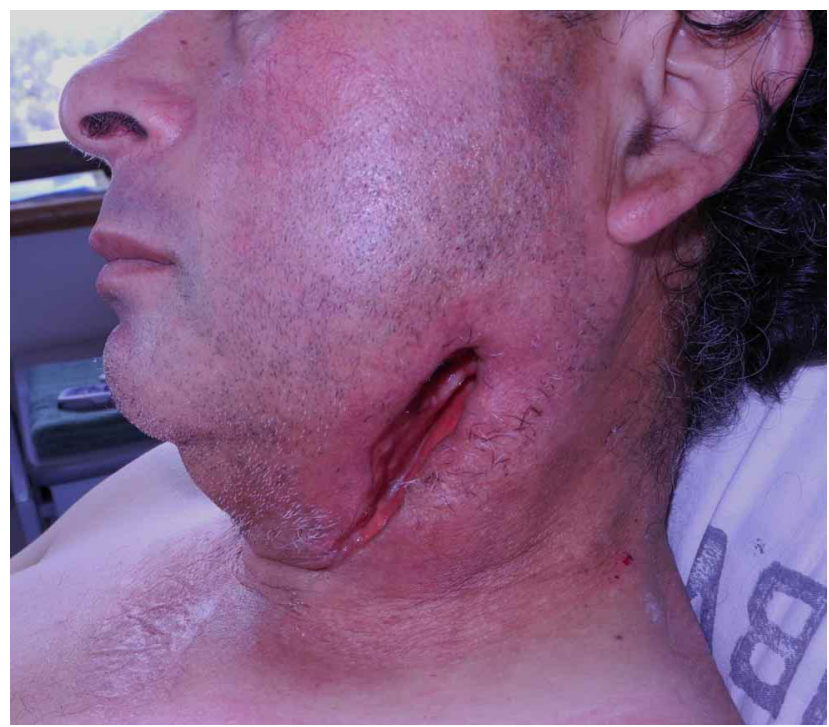

Fig. 5. Herida quirúrgica sin tejido necrótico, previo al alta del paciente.
La evolución de la herida siguió buen curso lográndose la regeneración de los tejidos desde sus planos profundos a sus planos superficiales, con la completa eliminación del tejido necrótico. El paciente acudió a la unidad de cirugía maxilofacial a control a los 3 meses posterior al alta hospitalaria, con correcta cicatrización de los bordes de la herida quirúrgica.

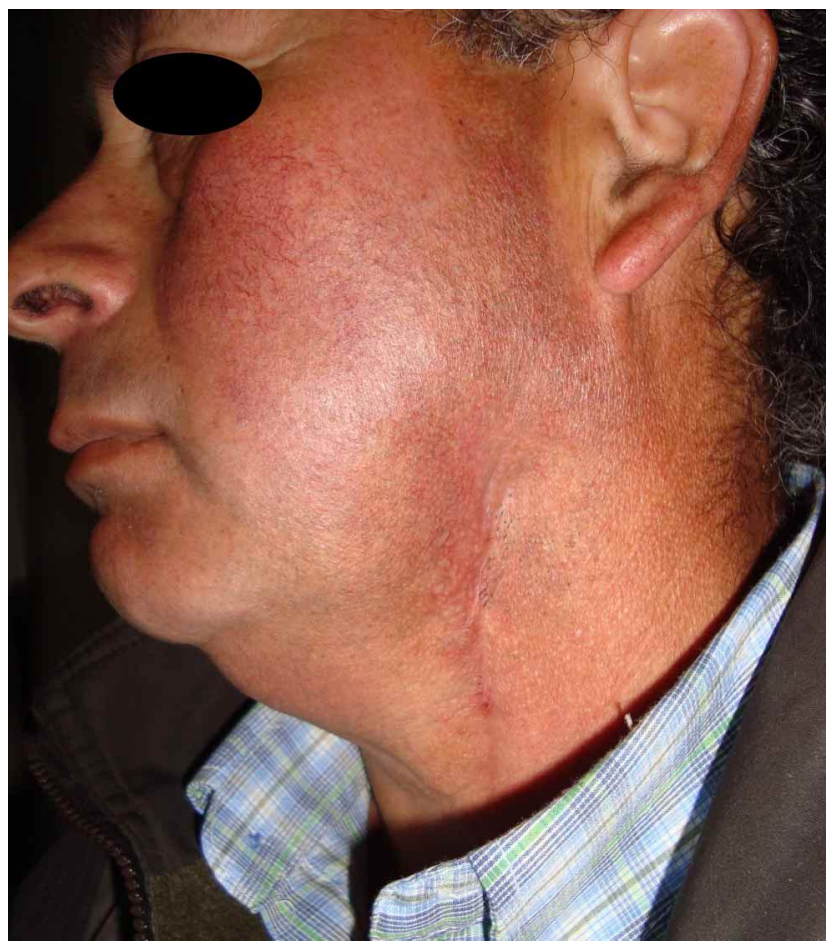

Fig. 6. Control a los 3 meses, presentando cicatriz remanente es de carácter umbilicada y lineal.

\section{DISCUSIÓN}

Los flegmones de cara y el cuello son las enfermedades infecciosas más graves de la región maxilofacial, pero también son menos comunes (Nienartowicz et al.).

El tratamiento tradicionalmente considera la incisión quirúrgica para el vaciamiento y drenaje, y el respectivo tratamiento antibiótico (Nienartowicz et al.). Varios son los estudios que confirman este tratamiento como efectivo en la resolución de las infecciones maxilofaciales. Wang et al. evaluaron 250 ingresos hospitalarios por infecciones maxilofaciales, de los cuales la mayoría requerían extracción, incisión, drenaje y antibióticos, siendo pocos los casos en que el cuadro se solucionaba sólo con antibióticos y extrac- 
ción. En este mismo sentido Akimbami et al. (2010) en un estudio retrospectivo, encontraron que en todos los casos de infecciones extendidas al tejido blando local/regional se realizó incisión y drenaje, además de la extracción.

Si bien las infecciones del área maxilofacial son un cuadro clínico habitual para los cirujanos maxilofaciales, la información relativa a los organismos encontrados en tales infecciones es escasa. Esto resulta en una situación paradójica porque, aunque la mayoría de los cirujanos maxilofaciales se ocupan de las infecciones maxilofaciales con buen manejo clínico, aún persiste un conocimiento vago e inadecuado de los organismos involucrados (Yuvaraj et al., 2012). Por esta razón es necesario presentar atención a una variedad de síntomas clínicos y considerando la posibilidad de exacerbación y remisión como en el caso presentado anteriormente (Nienartowicz et al.).

El papel de las bacterias anaeróbicas en las infecciones odontogénicas orofaciales está establecido, pero estudios recientes han sugerido que los cirujanos orales y maxilofaciales siguen teniendo puntos de vista tradicionales en lo relativo a la microbiología y el tratamiento de las infecciones odontogénicas (Yuvaraj et al.). Si bien existe en la literatura estudios recientes que no han encontrado cambios en la microflora conocida (Yuvaraj et al.) en casos como el presentado, que no tienen la evolución esperada con el tratamiento antibiótico empíri$\mathrm{co}$, se hace necesario realizar un cultivo de los tejidos afectados para establecer posibles variables en la flora microbiana asociada.

Existe una variedad de bacterias asociadas a infecciones maxilofaciales, estudios como el de Yuvaraj et al. en India señalaron que el Streptococcus y el Peptostreptococcus fueron las especies más encontradas de aerobios y anaerobios respectivamente.

Otros estudios como el de Cai et al. (2006), que evaluaron los microorganismos asociados a mediastinitis secundaria a infecciones cervicofaciales encontraron otros patógenos asociados como el Staphilococcus aureus meticilina resistentes, Enterobacter cloacae, Enterobacter aerogenes, Enterococcus faecalis, Streptococcus anginosus y Klebsiella pneumoniae. Esto concuerda con una de las bacterias encontradas en el caso, la enterobacter cloacae, que es un patógeno del tracto digestivo pero que también se ha asociado a heridas quirúrgicas, pero más bien se asocia a infecciones en pacientes inmunodeprimidos.

En este caso a pesar de que el paciente no presentaba patologías de base, requirió además del tratamiento en base a antibióticos y revulsivos locales, de dos intervenciones quirúrgicas para el drenaje y aseo quirúrgico. Debido al retraso en la correcta cicatrización de la herida quirúrgica se decidió la utilización de un hidrogel como terapia coadyuvante al tratamiento local.

Los hidrogeles son ampliamente utilizados como agentes de debridamiento en una variedad de heridas abiertas superficiales o profundas (por ejemplo, úlceras por presión, úlceras en piernas, heridas quirúrgicas y heridas malignas, quemaduras de espesor parcial, escaras y laceraciones). Son capaces de dar humedad al tejido deshidratado y absorber algo de humedad de heridas exudantes, además de proporcionar un ambiente húmedo para la curación de heridas. No son particulados, tóxicos ni adherentes, todas propiedades importantes si el lecho de la herida se debe mantener libre de dolor, bien hidratado y bien oxigenado. En general los hidrogeles tienen un efecto calmante en los tejidos y puede reducir significativamente el dolor en la herida (Jones \& Vaughan, 2005).

Un apósito ideal debe crear un ambiente húmedo para la cicatrización de heridas, absorber el exceso de exudado, permitir el intercambio gaseoso y ser fácilmente removido sin generar trauma a la herida (Wang et al.). En este sentido los hidrogeles se han vuelto cada vez más utilizados como apósitos para heridas debido a sus múltiples cualidades que incluyen la biocompatibilidad, relativamente bajo costo y capacidad para ser aplicado en diversas formas, tales como geles o sobre un apósito de gasa (Sheperd et al., 2011).

Diversos estudios han demostrado la eficacia

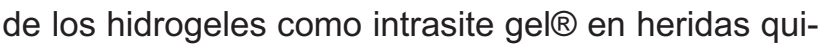
rúrgicas, coincidiendo en que es una medida costo/ efectiva, menos dolorosa y con menor trauma a los tejidos (Jones \& Vaughan).

En conclusión el manejo de cada caso de infección maxilofacial puede variar desde la terapia antibiótica hasta el manejo local de las heridas quirúrgicas, lo que concuerda con lo reportado en este caso, donde se logró la cicatrización completa en base a este hidrogel, sin producir una sobreinfección de la herida quirúrgica. 
VALENZUELA, A.; RAPOSO, A.; PREISLER, G. \& SALINAS, F. Management of surgical wound infection of maxillofacial area. Int. J. Odontostomat., 7(2):293-297, 2013.

ABSTRACT: The general objective in the management of mild or severe maxillofacial infections, is to protect the airway, realize surgical removal of the source of infection, surgical drainage of the lesion and adequate antibiotic therapy, control during onset of the lesion and facial injury until resolution the infectious process is complete. The aim of this paper is to present a case of an infection of maxillofacial area, difficult to manage locally and its response to treatment with development and management of the resulting facial wound. Patient is a 48 year-old male, without significant morbid history and is treated at the emergency department of the Base Hospital of Valdivia with a subsequent diagnosis of left facial phlegmon. During onset patient presented resistance to conventional treatment and wound healing was slow after emptying abscessed phlegmon. A hydrogel is used as an adjunct to therapy, leading finally to the resolution of clinical case. We discuss evolution of infectious event with various intervention therapies for severe infectious process.

KEY WORDS: maxillofacial infection, wound healing, wound dressing, hydrogel.

\section{REFERENCIAS BIBLIOGRÁFICAS}

Akinbami, B. O.; Akadiri, O. \& Gbujie, D. Spread of Odontogenic Infections in Port Harcourt, Nigeria. J. Oral Maxillofac. Surg., 68(10):2472-7, 2010.

Cai, X. Y.; Zhang, W. J.; Zhang, Z. Y.; Yang, C.; Zhou, L. N. \& Chen, Z. Cervical infection with descending mediastinitis: a review of six cases. Int. J. Oral Maxillofac. Surg., 35(11):1021-5, 2006.

Guerra, R.; Pérez, P.; Roque, R.; Bomant, E.; González, Y. \& Palenzuela, T. Efectividad del adhesivo tisular Tisuacryl en el cierre de heridas cutáneas. Rev. Cubana Med. Gen. Integr., 21(1-2):1-10, 2005.

Jones, A. \& Vaughan, D. Hydrogel dressings in the management of a variety of wound types: A review. J. Orthop. Nurs., 9(1):S1-11, 2005.

Nienartowicz, J.; Gerber, H.; Pawlak, W. \& Wnukiewicz, J. Phlegmon of the face and neck - own observations. Czas. Stomatol., 61(2):106-13, 2008.

Ramírez, R. \& Dagnino, B. Curación de heridas. Antiguos conceptos para aplicar y entender su manejo avanzado. Cuad. Cir., 20(1):92-9, 2006.

Shepherd, J.; Rimmer, S.; Swanson, L.; MacNeil, S. \& Douglas, I. Hyperbranched poly(NIPAM) polymers modified with antibiotics for the reduction of bacterial burden in infected human tissue engineered skin. Biomaterials., 32(1):258-67, 2011.

Tejeda, E.; Rodríguez, J. \& Gayosso, O. Abscesos profundos de cuello. Reporte de cinco casos y revisión de la literatura. Rev. Asoc. Mex. Med. Crit. Ter. Int., 13(4):142-5, 1999.

Wang, T.; Zhu, X.; Xue, X. \& Wu, D. Hydrogel sheets of chitosan, honey and gelatin as burn wound dressings. Carbohydr. Polymer., 88(1):75-83, 2012.
Yuvaraj, V.; Alexander, M. \& Pasupathy, S. Microflora in maxillofacial infections--a changing scenario? J. Oral Maxillofac. Surg., 70(1):119-25, 2012.

Dirección para Correspondencia:

Dra. Araceli Raposo Castillo

Departamento de Cirugía Buco-maxilofacial

Universidad Austral de Chile

Valdivia

CHILE

Email: araceliraposo@gmail.com

Recibido : 26-07-2012

Aceptado: 22-03-2013 